\title{
The Collaborative Ocular Tuberculosis Study (COTS)-1: A Multinational Review of 165 Patients with Tubercular Anterior Uveitis
}

\section{Authors:}

Rupesh AGRAWAL ${ }^{1,2,3,4^{*}}$, Bjorn BETZLER ${ }^{1,4}$, llaria TESTI ${ }^{3}$, Sarakshi MAHAJAN5 Aniruddha AGARWAL ${ }^{6}$, Dinesh Visva GUNASEKERAN ${ }^{2,3}$, Dhananjay RAJE ${ }^{7}$, Kanika AGGARWAL ${ }^{6}$, Somasheila I MURTHY ${ }^{8}$, Mark WESTCOTT ${ }^{3}$, Soon-Phaik CHEE $^{1,4,9,10}$, Peter MC CLUSKEY ${ }^{11}$, Su Ling $\mathrm{HO}^{2}$, Stephen TEOH ${ }^{2}$, Luca CIMINO ${ }^{12}$, Jyotirmay BISWAS $^{13}$, Shishir NARAIN ${ }^{14}$, Manisha AGARWAL ${ }^{15}$, Padmamalini MAHENDRADAS ${ }^{16}$, Moncef KHAIRALLAH $^{17}$, Nicholas JONES ${ }^{18}$, Ilknur TUGALTUTKUN $^{19}$, Kalpana BABU ${ }^{20}$, Soumayava BASU ${ }^{21}$, Ester CARREÑO ${ }^{22}$, Richard LEE ${ }^{3}$, Hassan AL-DHIBI ${ }^{23}$, Bahram BODAGHI ${ }^{24}$, Alessandro INVERNIZZI ${ }^{25}$, Debra A. GOLDSTEIN $^{26}$, Carl P. HERBORT ${ }^{27}$, Talin BARISANI-ASENBAUER ${ }^{28}$, Julio J GONZÁLEZ-LÓPEZ ${ }^{29}$, Sofia ANDROUDI ${ }^{30}$, Reema BANSAL ${ }^{6}$, Bruttendu MOHARANA $^{6}$, Simona ESPOSTI ${ }^{3}$, Anastasia TASIOPOULOU ${ }^{3}$, Sengal NADARAJAH ${ }^{3}$, Mamta AGARWAL ${ }^{13}$, Sharanaya ABRAHAM $^{13}$, Ruchi VALA $^{16}$, Ramandeep SINGH ${ }^{6}$, Aman SHARMA ${ }^{31}$, Kusum SHARMA ${ }^{32}$, Manfred ZIERHUT ${ }^{33}$, Onn Min KON ${ }^{34}$, Emmett T. CUNNINGHAM ${ }^{35}$, John H. KEMPEN ${ }^{36,37}$, Quan Dong NGUYEN ${ }^{5}$, Carlos PAVESIO ${ }^{3}$, Vishali GUPTA ${ }^{6}$.

\section{Affiliations}

1. Yong Loo Lin School of Medicine, National University of Singapore, Singapore

2. National Healthcare Group Eye Institute, Tan Tock Seng Hospital, Singapore

3. Moorfields Eye Hospital, NHS Foundation Trust, London, United Kingdom

4. Singapore Eye Research Institute, Singapore

5. Byres Eye Institute, Stanford University, Palo Alto, California, United States of America

6. Advanced Eye Centre, Postgraduate Institute of Medical Education and Research (PGIMER), Chandigarh

7. MDS Bioanalytics, India

8. Tej Kohli Cornea Institute, LV Prasad Eye Institute, Kallam Anji Reddy Campus, Hyderabad, India

9. Singapore National Eye Centre, Singapore

10. Department of Ophthalmology \& Visual Sciences Academic Clinical Program, Duke-NUS Medical School

11. Department of Clinical Ophthalmology \& Eye Health, Central Clinical School, Save Sight Institute, The University of Sydney, Sydney, Australia

12. Ocular Immunology Unit, Azienda USL IRCCS, Reggio Emilia, Italy

13. Department of Ophthalmology, Sankara Nethralaya, Chennai, India

14. Department of Ophthalmology, Shroff Eye Centre, New Delhi, India

15. Department of Ophthalmology, Dr Shroff's Charity Eye Hospital Daryaganj, New Delhi, India

16. Department of Uveitis and Ocular Immunology, Narayana Nethralaya, Bangalore, India

17. Department of Ophthalmology, Fattouma Bourguiba University Hospital, Faculty of Medicine, University of Monastir, Tunisia

18. Department of Ophthalmology, University of Manchester, Manchester, United Kingdom

19. Istanbul University, Istanbul Faculty of Medicine, Department of Ophthalmology, Turkey

20. Prabha Eye Clinic \& Research centre, Vittala International Institute of Ophthalmology, Bangalore, India

21. LV Prasad Eye Institute, Department of Ophthalmology, Bhubaneswar, India

22. Hospital Universitario Fundacion Jimenez Diaz, Madrid, Spain

23. King Khaled Eye Specialist Hospital, Department of Ophthalmology, Riyadh, Kingdom of Saudi Arabia 
24. DHU SightRestore, Department of Ophthalmology, Sorbonne University, Paris, France

25. Eye Clinic, Department of Biomedical and Clinical Science "L. Sacco", Luigi Sacco Hospital, University of Milan, Italy

26. Northwestern University, Feinberg School of Medicine, Department of Ophthalmology. Chicago, Illinois, USA

27. Centre for Ophthalmic Specialised Care \& University of Lausanne, Lausanne, Switzerland

28. The Centre for Ocular Inflammation and Infection (OCUVAC), Laura Bassi Centre of Expertise Center of Pathophysiology, Infectiology and Immunology, Medical University of Vienna, Vienna, Austria

29. Ramón y Cajal University Hospital, Department of Ophthalmology, Madrid, Spain

30. Department of Ophthalmology, University of Thessaly, Greece

31. Department of Rheumatology, PGIMER, Chandigarh, India

32. Department of Microbiology, PGIMER, Chandigarh, India

33. Centre of Ophthalmology, Department of Ophthalmology, University of Tuebingen, Germany

34. Chest and Allergy Clinic, St Mary's Hospital, Imperial College Healthcare NHS Trust, London, United Kingdom

35. The Francis I. Proctor Foundation, UCSF School of Medicine, San Francisco, California, United States

36. Department of Ophthalmology, Massachusetts Eye and Ear, Harvard Medical School, Boston, Massachusetts, United States

37. MCM Eye Unit, MyungSung Christian Medical Center and MyungSung Medical School, Addis Ababa, Ethiopia

\section{Corresponding author:}

Vishali Gupta, MD

Professor of Ophthalmology,

Advanced Eye Centre,

Post graduate Institute of Medical Education and Research (PGIMER),

Chandigarh, India.

Email: vishalisara@yahoo.co.in

Contact number: +919417565506 


\section{ABSTRACT}

Purpose: The Collaborative Ocular Tuberculosis Study (COTS) Group sought to address the diagnostic uncertainty through retrospective cohort analysis of treatment regimens and therapeutic outcomes for patients with tubercular Anterior Uveitis (TAU) across international centres.

Methods: Multicentre retrospective analysis of patients diagnosed with TAU between January 2004 to December 2014 that had a minimum follow-up of 1 year.

Results: One hundred and sixty-five patients were included. One hundred and seven subjects received antitubercular therapy (ATT) ( $n=107 / 165 ; 64.9 \%$ ) with all the patients receiving topical steroid therapy. Treatment failure was noted in 17 patients $(n=17 / 165 ; 10.3 \%)$, more frequently described in patients that received ATT $(n=$ $13 / 107,12.2 \%)$, than those that did not receive ATT $(n=4 / 58,6.9 \%)$.

Conclusion: In this retrospective study, addition of ATT did not have any statistically significant impact on outcome in patients with TAU.

Keywords: Anti-tubercular therapy; tubercular anterior uveitis; ocular tuberculosis; uveitis; Collaborative Ocular Tuberculosis Study (COTS) 


\section{INTRODUCTION}

Tuberculosis (TB) is one of the most significant global diseases with a substantial burden on healthcare systems worldwide, with the World Health Organization estimating that TB costs the world over $\$ 21$ billion each year collectively. ${ }^{1}$ The large pool of latent Mycobacterium tuberculosis (Mtb) contributes to the difficulty of its eradication. It is estimated that around a third of the global population has been infected by Mtb, and most of them are asymptomatic. ${ }^{2}$ When active, Mtb may disseminate haematogenously from the pulmonary tissue, resulting in extrapulmonary manifestations. Ocular Tuberculosis (OTB) is one of the extrapulmonary manifestations of the disease. Reports on its incidence and prevalence vary amongst global centres due to epidemiological differences. ${ }^{3}$ Estimates from cross-sectional studies place TB incidence in non-endemic regions, such as Europe and USA, at 1\% - $4 \%$, while it might be as high as $10 \%-26 \%$ in highly endemic regions, such as India and Saudi Arabia. ${ }^{4}$

Uveitis is the most common presentation of OTB. Gupta et al. described the distribution of uveal involvement in patients with a presumptive diagnosis of tubercular uveitis (TBU). ${ }^{5}$ Out of 182 participants, $70(39 \%)$ had posterior uveitis, $53(29 \%)$ had anterior uveitis, 29 (16\%) had intermediate uveitis, and $30(16 \%)$ had panuveitis.

The diagnosis of OTB is usually presumptive, made empirically in the presence of corroborating features, exclusion of differential diagnoses, and physician expertise taking into account the local epidemiology. 6,7 The traditional time-consuming method of culturing ocular fluid-derived samples for the presence of $M$. tuberculosis remains the only unambiguous clinical diagnostic test of active TB to date, with associated risks given that it is an invasive test. Furthermore, clinicians face difficulties when electing to use other tests, such as Interferon Gamma Release Assays (IGRA), Mantoux Skin Tests, chest X-Rays, and polymerase chain reaction (PCR) assays given variable performance of these tests in terms of sensitivity and specificity for the detection of TB. 3,5,6,8-10 The combination of nonspecific clinical manifestations of OTB and the lack of definitive diagnostic tests have led to differences in clinical decisions on when to initiate anti-tubercular therapy (ATT) for OTB patients, and how regimens should vary based on specific clinical phenotype. Since the infection itself is often challenging to 
detect, most literature has highlighted a diagnostic uncertainty and an inability to propose proven treatment guidelines for OTB.

The Collaborative Ocular Tuberculosis Study (COTS) - 1 was proposed to enhance the study of clinical management and treatment outcomes of OTB. COTS- 1 is a multicentre retrospective cohort study of patients with OTB, designed to facilitate the compilation and analysis of presenting clinical features of TBU and observed treatment outcomes from multiple centres worldwide. It grants an overview of how the disease currently is approached internationally.

As recently defined by the COTS Nomenclature Working Group, tubercular anterior uveitis (TAU) is a subgroup of OTB of which inflammation has been limited to the anterior segment, involving the iris and ciliary body, in accordance with classification by the Standardization of Uveitis Nomenclature (SUN) Working Group. ${ }^{11}$ Here we describe the observed outcomes of patients with TAU from the recently concluded COTS -1 .

\section{METHODS}

COTS -1 is a retrospective cohort study of patients diagnosed with OTB, conducted from January 2004 to December 2014 across 25 multinational centres in 14 countries. $^{7}$ Demographics, ocular phenotypes, investigations, management, and treatment outcomes were extracted from medical records. Participating centres sought ethical approval from their respective local institutional ethics committees. Participating centres are listed in Appendix 1. Diagnostic criteria for OTB were established based on the presence of suggestive clinical features and corroborative results of investigations. Appendix 2 lists the inclusion criteria used by the COTS Study Group. In brief, the criteria include:

1. Satisfied study diagnostic criteria for TAU.

2. Availability of patient medical records detailing baseline ophthalmic examination and follow-up reviews

3. Ancillary and laboratory investigations done to exclude relevant differential diagnoses.

4. A minimum follow-up period of 1 year was completed. 
Patients who satisfied all the above inclusion criteria were enrolled in the study. Following which, the diagnosis of TAU was conferred to patients who fulfilled these criteria and had any form of involvement of the anterior uveal tract upon initial presentation. Classification of the anterior phenotype was established by the attending uveitis specialist ophthalmologist. There was non-informative right censoring as the follow-up interval was capped at 2 years regardless of treatment response. Incomplete follow-up in some patients was related to transfer to other centres, death, and other losses to follow-up.

Treatment regimen with ATT and/or immunosuppression was guided by attending physicians in collaboration with respiratory or infectious disease specialists as per respective institutional protocols. The route of drug delivery for corticosteroids and use of corticosteroid-sparing immunosuppressive agents was decided by the attending uveitis specialist ophthalmologist on a case-by-case basis guided by clinical phenotype, severity of OTB, patients' comorbidities, and treatment response.

Treatment failure for individual patients during follow-up was determined using criteria based on the treatment regimen received. Treatment failure was defined as any of the following:

1. Persistence or recurrence of inflammation within 6 months of completing ATT.

2. Inability to taper oral corticosteroids to $<10 \mathrm{mg} /$ day or corticosteroid eye drops to less than 2 drops daily, without recurrence of inflammation.

3. Recalcitrant inflammation necessitating corticosteroid-sparing immunosuppressive therapy.

\section{Data Collection}

A novel data entry platform was developed to address the heterogeneous nature of this disease. The variable of treatment failure was assessed at standardized 6 monthly time intervals of follow-up from initial diagnosis: 6 months, 12 months, 18 months, and 24 months. Variables for which data were not entered were treated as missing values with pairwise deletion for statistical analysis.

\section{Statistical Analysis}

Frequencies and percentages were calculated for different study variables. The categorical variables were compared using Pearson's $x^{2}$ test, while continuous variables were compared using $t$-test for independent samples. Non-parametric 
survival analysis with Kaplan-Meier plots was performed to determine the uveitis recurrence-free survival patterns in 2 groups - ATT administered and ATT nonadministered. The statistical significance of the difference in survival rates was determined using the Log-rank test.

Linear mixed modelling was performed to determine if visual acuity differed significantly between ATT administered and non-administered groups over time. The fixed effects were treatment administered (ATT or no ATT) and sex. Patient ID was regarded as a random effect. The unstructured variance-covariance relationship was considered for visual acuity across time. All analyses were conducted using SPSS statistical software (IBM SPSS Statistics for Windows, Version 25.0. Armonk, NY: IBM Corp.); statistical significance was tested at 5\% type 1 error probability without adjustment for multiple comparisons (as recommended for observational studies). ${ }^{12}$

\section{RESULTS}

One hundred and sixty-five patients with TAU were included in the study. Patients had a mean age of $45.4 \pm 15.7$ years. Females represented $52.7 \%$ of this cohort $(n=$ $87 / 165)$ and $63 \%$ were of Asian geographical origin $(n=104 / 165)$. Around $31 \%(n=$ $52 / 165)$ of the patients had chest features consistent with inactive or healed pulmonary TB, in particular 42/165 (25.4\%) and 10/165 (6.0\%) had positive findings on chest Xray and chest computed tomography, respectively. The majority of subjects presented with normal visual acuity ( $n=102 / 165,69.4 \%$ ). Demographics and the clinical presentations of patients are further described in Table 1.

Table 2 compares characteristics of patients separated into two groups according to ATT administration. Overall, 107 patients received ATT ( $n=107 / 165 ; 64.9 \%)$ and all the patients had topical steroid therapy (with different dosage, varying duration and varying number of times). Some of the patients with severe recurrent anterior uveitis $(n=23 / 165 ; 13.93 \%)$ had short courses of oral steroids as well but the details for duration and dosage was not recorded in the data entry form. Treatment failure occurred in 17 patients ( $\mathrm{n}=17 / 165 ; 10.3 \%$ ), more frequently those who had received ATT $(n=13 / 107,12.2 \%)$, than those not treated with ATT $(n=4 / 58,6.9 \%)$. However, this difference was not statistically significant $(p=0.43)$. Except for laterality of presentation ( $p=0.045$ ), all other characteristics also reflected statistically insignificant differences between the two groups ( $p>0.05)$. 
Figure 1 shows the Kaplan-Meier plots obtained when survival analysis was conducted in this cohort of 165 patients with TAU. The survival analysis was conducted based on 'Time to treatment failure', and compares the group that received ATT with the group that did not receive ATT. Among patients who were treated with ATT, the mean recurrence-free survival time was 21.0 months (95\% Cl: $19.3-22.7$ ). The group that did not receive ATT had a longer mean recurrence-free survival time of 22.5 ( $95 \% \mathrm{Cl}: 20.6$ - 24.4) months, but the difference was not statistically significant based on the Log-rank test ( $p$-value $=0.37$ ).

Figure 2 compares the mean visual acuity score (in Snellen decimals) of the ATTtreated and -untreated groups over time. Visual acuity was measured at 0, 3, 6, 12, 18 and 24 months. For statistical reasons, the visual acuity was expressed in Snellen's decimal. So a VA of 1.0 indicate normal vision, while 0.0 indicate vision loss. After adjusting for gender and age, mean visual acuity for patients that received ATT was 0.73 (95\% Cl: $0.52-0.93$ ), while that for patients that did not receive ATT was 0.65 $(95 \% \mathrm{Cl}: 0.56-0.74)$. The difference was statistically insignificant ( $p$-value $=0.20)$.

\section{DISCUSSION}

The study provides information on patients with TAU and the corresponding outcomes of ATT treatment. Contrary to our expectations, we found that treatment failure occurred similarly often among ATT-treated and untreated individuals. Visual acuity outcomes also were similar. We hypothesise that potential explanations of the lack of benefit from ATT could be attributed to an association with latent TB rather than causality in some cases of TAU, or to TB contribution to initiating but not to maintaining the chronic inflammatory process. Current literature does highlight that OTB overdiagnosis is a problem faced by clinicians due to diagnostic tools which are not unambiguously confirmative. ${ }^{3,13,14}$ Drug-drug interactions, such as the reduced tissue bioavailability and increased plasma clearance of prednisolone in concurrent administration with rifampicin, could play a role too. ${ }^{15}$ Furthermore, drug sensitivity of the presenting OTB strain was not established in most patients, and is not typically done because of the low yield of ocular-fluid derived cultures, long timeframe, and associated risks. ${ }^{16,17}$ In TB endemic regions, physicians could be more inclined to initiate rather than withhold ATT because of a greater likelihood of a tuberculous aetiology. ${ }^{14,18}$ This is not entirely unwarranted because untreated OTB could place the 
patient at risk of high visual morbidity. ${ }^{7,19}$ However, our results suggest that this practice is not beneficial in preventing TAU recurrence or in improving visual results. It is an ongoing hypothesis that a predominant presentation of anterior uveitis in OTB represents an ocular manifestation of autoimmune reactivity to molecular mimicry of latent TB, rather than direct infection of the uvea with active TB seeded from pulmonary foci. ${ }^{7,20}$ In this case, a primarily anti-inflammatory regimen should be used. The difficulty arises because conventional immunologic tests used in TB uveitis cannot distinguish between these two possible mechanisms of disease pathogenesis. Future studies exploring the differentiation between para-infectious autoimmune and infectious causes of TAU could thus be useful in guiding more accurate implementation of corticosteroid and ATT regimens.

As part of the COTS-1 study, manuscripts have been published analysing the patterns of ATT treatment and clinical outcomes among patients with tubercular retinal vasculitis and with choroidal involvement. ${ }^{21,22}$ Fewer TAU cases used ATT ( $\mathrm{n}=$ $107 / 165,64.8 \%$ ) than we observed for cases of tubercular retinal vasculitis ( $\mathrm{n}=$ $228 / 251,90.8 \%$ ) and the subset analysing choroidal involvement ( $n=219 / 245$, $89.38 \%$ ). ${ }^{21}$ The individual reasons for choosing or not choosing ATT could not be analysed because of the lack of documented rationale and the retrospective nature of this study. Nevertheless, some possible reasons include patient reluctance because of lack of strong robust studies demonstrating efficacy of ATT in preventing recurrences of inflammation in TAU and adequacy of topical corticosteroids in management, as determined by the attending physicians. Also, the absolute risk of recurrence/treatment failure was low, which may raise the threshold for ATT treatment in TAU. In the present study, a significantly $(p=0.045)$ higher proportion of patients presented with unilateral TAU (compared to bilateral TAU) in the group not treated with ATT $(n=42 / 58,72.4 \%$ ) than in the group treated with ATT ( $n=59 / 107,55.1 \%$ ). The patients not treated with ATT had better vision with time as the mean scores are higher that those treated with ATT for the follow up times, except at presentation (Table 2, Figure 2). The vision deterioration is at a lesser rate in non-treated group as compared to treated group.

Although from this retrospective analysis it appeared that the addition of ATT to topical therapy does not significantly affect the treatment outcome of TAU patients, different 
single centers studies reported a therapeutic role of ATT in patients with TAU. 2, 6, 23, 24, 26-39 In addition, from COTS consensus guidelines (unpublished data) it emerged that experts are keen to start ATT in TAU in case of recurrent episodes in patients with one immunological evidence of TB, together with radiological findings suggestive of active or healed pulmonary TB, irrespective of patient's endemicity. In case of first episode of TAU, experts are inclined to initiate ATT in patients with both immunological and radiological tests positive, coming from both endemic and non endemic region.

A limitation for this study is its retrospective methodology and the resultant unstandardized clinical definition and diagnostic criteria of TAU. Methods of documentation also varied across the multinational centres, and this has led to missing data, like the one related to the cause of visual impairment in patients with anterior uveitis. Furthermore, almost $90 \%$ of the patients came from the northern hemisphere and there were no data from South America and sub-equatorial Africa, with small numbers of patients in some categories, such as those coming from Middle-Eastern region. Another point to note is that owing to the lack of a global standardised criteria for the definitive diagnosis of TBU, the 165 cases in this study can best be described as anterior uveitis with a presumed tubercular aetiology. That being said, the diagnosis of TAU was sufficiently arrived at by attending uveitis experts in consultation with infectious disease specialists when indicated; this involved the consideration of clinical, immunological, and radiological criteria, and the exclusion of other infectious and non-infectious differentials.

That being said, the strengths of this study must be acknowledged. This paper, part of the COTS-1 study, describes the only multinational dataset of TAU to date based on a large and diverse patient cohort. Inclusion, exclusion and diagnostic criteria have been standardized so as to address the limitations of the existing literature on TAU. For instance, current reports of TAU are retrospective studies in isolated centres. ${ }^{3,13,23-}$ 26 Cross comparison between these reports is difficult because of inconsistencies in inclusion criteria and varying methods of outcomes analysis. Future studies can build on the current results through prospective methodology, mandatory documentation of key clinical features, and proper explanation of the reasons why some patients are not started on ATT by attending physicians.

\section{CONCLUSION}


The current study provides information from multinational centres on patients presenting with TAU, and the corresponding outcomes of ATT treatment. The (absolute) risk of relapse is low and it doesn't differ based on ATT treatment as far we can see taking into account the limitations of the retrospective, non-randomized design. Limitations with respect to the retrospective nature of the study, among others, imply that the current study cannot provide conclusive evidence on the therapeutic benefit of ATT in TAU. One of the inferences from this study can be that if the risk of relapse is low anyway, and probably the risks of vision loss with relapse promptly treated also is low, then we may not consider initiating ATT in patients with TAU. This study highlights the real need for the design of systematic and global collaborative prospective studies to advance the current understanding of the TAU and its management. 


\section{DECLARATION OF INTEREST}

The authors report no conflicts of interest, financial or non-financial, in the subject matter or materials discussed in this manuscript. Prof Kempen is consultant for Gilead (DSMC chair) and also equity owner for Betaliq. The work was supported by NIHR biomedical research, UK. The authors alone are responsible for the content and writing of the paper.

\section{Acknowledgements:}

The research was partially funded by the National Institute for Health Research (NIHR) Biomedical Research Centre at Moorfields Eye Hospital NHS Foundation Trust and UCL Institute of Ophthalmology. This sponsor supported some of the research manhours that were contributed by all our part-time collaborators from the Moorfields Eye Hospital that are salaried as Ophthalmology clinicians by the hospital. Debra A. Goldstein is supported by an unrestricted grant from Research to Prevent Blindness (RPB). Vishali Gupta is supported by grant from Department of Biotechnology (DBT), India. Julio J GONZÁLEZ-LÓPEZ2 is supported by study grants from Abbvie, Allergan, and Angelini. Funders played no part in the design and conduct of the study; collection, management, analysis, and interpretation of the data; preparation, review, or approval of the manuscript; nor the decision to submit the manuscript for publication. Rupesh AGRAWAL, Dinesh Visva GUNASEKERAN, and Dhananjay RAJE had full access to all the data in the study and takes responsibility for the integrity of the data and the accuracy of the data analysis. 


\section{BIBLIOGRAPHY}

1. Dheda K, Barry CE 3rd, Maartens G. Tuberculosis. Lancet. 2016;387(10024):1211-26.

2. Conant MM, Vrasich CR, Wongskhaluang JV, et al. Role of the Infectious Disease Consultant in Management of Patients With Tuberculosis-Associated Ocular Inflammation. Open Forum Infect Dis. 2015;3(1):ofv195.

3. Agrawal R, Gunasekeran DV, Raje D, et al.Collaborative Ocular Tuberculosis Study Group. Global Variations and Challenges With Tubercular Uveitis in the Collaborative Ocular Tuberculosis Study. Invest Ophthalmol Vis Sci. 2018;59(10):4162-4171.

4. Abu El-Asrar AM, Abouammoh M, Al-Mezaine HS. Tuberculous uveitis. Int Ophthalmol Clin. 2010;50(2):19-39.

5. Gupta B, Agrawal R, Swampillai AJ, et al. Ocular manifestations of tuberculosis: an update. Expert Rev Ophthalmol. 2016;11(2):145-54.

6. Bansal R, Gupta A, Gupta V, Dogra MR, Bambery P, Arora SK. Role of anti-tubercular therapy in uveitis with latent/manifest tuberculosis. Am J Ophthalmol. 2008;146(5):772-9.

7. Agrawal R, Gunasekeran DV, Grant R, et al. Collaborative Ocular Tuberculosis Study (COTS)1 Study Group. Clinical Features and Outcomes of Patients With Tubercular Uveitis Treated With Antitubercular Therapy in the Collaborative Ocular Tuberculosis Study (COTS)-1. JAMA Ophthalmol. 2017;135(12):1318-1327.

8. Ang M, Chee SP. Controversies in ocular tuberculosis. Br J Ophthalmol. 2017;101(1):6-9.

9. Agarwal A, Agrawal R, Gunasekaran DV,et al. The Collaborative Ocular Tuberculosis Study (COTS)-1 Report 3: Polymerase Chain Reaction in the Diagnosis and Management of Tubercular Uveitis: Global Trends. Ocul Immunol Inflamm. 2019;27(3):465-473.

10. Ang M, Htoon HM, Chee SP. Diagnosis of tuberculous uveitis: clinical application of an interferon-gamma release assay. Ophthalmology. 2009;116(7):1391-6.

11. Jabs DA, Nussenblatt RB, Rosenbaum JT; Standardization of Uveitis Nomenclature (SUN) Working Group. Standardization of uveitis nomenclature for reporting clinical data. Results of the First International Workshop. Am J Ophthalmol. 2005;140(3):509-16.

12. Wang R, Lagakos SW, Ware JH, Hunter DJ, Drazen JM. Statistics in medicine-reporting of subgroup analyses in clinical trials. N Engl J Med. 2007;357(21):2189-94

13. Kee AR, Gonzalez-Lopez JJ, Al-Hity A, et al.Anti-tubercular therapy for intraocular tuberculosis: A systematic review and meta-analysis. Surv Ophthalmol. 2016;61(5):628-53.

14. Basu S, Nayak S, Padhi TR, Das T. Progressive ocular inflammation following anti-tubercular therapy for presumed ocular tuberculosis in a high-endemic setting. Eye (Lond). 2013;27(5):65762. 
15. McAllister WA, Thompson PJ, Al-Habet SM, Rogers HJ. Rifampicin reduces effectiveness and bioavailability of prednisolone. Br Med J (Clin Res Ed). 1983;286(6369):923-925.

16. Pantoja A, Kik SV, Denkinger CM. Costs of novel tuberculosis diagnostics--willcountries be able to afford it? J Infect Dis. 2015;211 Suppl 2:S67-77.

17. Zanetti S, Bua A, Molicotti P, Maiore I, Pinna A. QuantiFERON-TB Gold Assay on Plasma for Confirmation of Presumed Tuberculosis-Related Uveitis. J Clin Microbiol. 2016;54(8):2175-7.

18. Singh R, Gupta V, Gupta A. Pattern of uveitis in a referral eye clinic in north India. Indian J Ophthalmol. 2004;52(2):121-5.

19. Gunasekeran DV, Gupta B, Cardoso J, Pavesio CE, Agrawal R. Visual Morbidity and Ocular Complications in Presumed Intraocular Tuberculosis: An Analysis of 354 Cases from a NonEndemic Population. Ocul Immunol Inflamm. 2018;26(6):865-869.

20. Spratt A, Key T, Vivian AJ. Chronic anterior uveitis following bacille Calmette-Guérin vaccination: molecular mimicry in action? J Pediatr Ophthalmol Strabismus. 2008;45(4):252-3.

21. Gunasekeran DV, Agrawal R, Agarwal A, et al. COTS-1 Study Group. THE COLLABORATIVE OCULAR TUBERCULOSIS STUDY (COTS)-1: A Multinational Review of 251 Patients With Tubercular Retinal Vasculitis. Retina. 2019;39(8):1623-1630.

22. Agrawal R, Gunasekeran DV, Agarwal A, et al. The Collaborative Ocular Tuberculosis Study (COTS)-1: A Multinational Description of the Spectrum of Choroidal Involvement in 245 Patients with Tubercular Uveitis. Ocul Immunol Inflamm. 2018;29:1-11.

23. Al-Qarni A, Abouammoh MA, Almousa AN, Mousa A, Abu El-Asrar AM. Presumed tuberculous uveitis in a university-based tertiary referral center in Saudi Arabia. Int Ophthalmol. 2019;39(2):317-333.

24. Ang M, Hedayatfar A, Wong W, Chee SP. Duration of anti-tubercular therapy in uveitis associated with latent tuberculosis: a case-control study. Br J Ophthalmol. 2012;96(3):332-6.

25. Ang L, Kee A, Yeo TH, et al. Treatment outcome in patients with presumed tubercular uveitis at a tertiary referral eye care centre in Singapore. Int Ophthalmol. 2018;38(1):11-18.

26. Khochtali S, Gargouri S, Abroug N, et al. The spectrum of presumed tubercular uveitis in Tunisia, North Africa. Int Ophthalmol. 2015;35(5):663-71.

27. Ng KK, Nisbet M, Damato EM, Sims JL. Presumed tuberculous uveitis in non-endemic country for tuberculosis: case series from a New Zealand tertiary uveitis clinic. Clin Exp Ophthalmol. 2017 May;45(4):357-365.

28. Agrawal R, Grant R, Gupta B et al. What does IGRA testing add to the diagnosis of ocular tuberculosis? A Bayesian latent class analysis. BMC Ophthalmol. 2017;17(1):245.

29. Babu K, Satish V, Prakash O, Subbakrishna DK, Murthy KR. Role of the mantoux test and treatment with antitubercular therapy in a South Indian patient population of presumed intraocular tuberculosis. Ocul Immunol Inflamm. 2009;17(5):307-11. 
30. Ahn SJ, Kim KE, Woo SJ, Park KH. The usefulness of interferon-gamma release assay for diagnosis of tuberculosis-related uveitis in Korea. Korean J Ophthalmol. 2014;28(3):226-33.

31. Babu K, Satish V, Satish S, Subbakrishna DK, Abraham MP, Murthy KR. Utility of QuantiFERON TB gold test in a south Indian patient population of ocular inflammation. Indian $\mathrm{J}$ Ophthalmol. 2009;57(6):427-30.

32. Campos WR, Henriques JF, Kritski AL, Curi A, Pimentel RT, Spindola de Miranda S. Tuberculous uveitis at a referral center in southeastern Brazil. J Bras Pneumol. 2008;34(2):98102.

33. Chung CY, Li KKW. The efficacy of latent tuberculosis treatment for immunocompetent uveitis patients with a positive T-SPOT.TB test: 6-year experience in a tuberculosis endemic region. Int Ophthalmol. 2018;38(6):2273-2282.

34. Shahidatul-Adha M, Zunaina E, Liza-Sharmini AT, et al. Ocular tuberculosis in Hospital Universiti Sains Malaysia - A case series. Ann Med Surg (Lond). 2017;24:25-30.

35. Agrawal R, Gonzalez-Lopez JJ, Nobre-Cardoso J, et al. Predictive factors for treatment failure in patients with presumed ocular tuberculosis in an area of low endemic prevalence. $\mathrm{Br} J$ Ophthalmol. 2016;100(3):348-55.

36. Cimino L, Herbort CP, Aldigeri R, Salvarani C, Boiardi L. Tuberculous uveitis, a resurgent and underdiagnosed disease.. Int Ophthalmol. 2009;29(2):67-74.

37. Damato EM, Dawson S, Liu X, Mukherjee C, et al. A retrospective cohort study of patients treated with anti-tuberculous therapy for presumed ocular tuberculosis. J Ophthalmic Inflamm Infect. 2017;7(1):23.

38. Jakob E, Max R, Zimmermann S, et al. Three years of experience with QuantiFERON-TB gold testing in patients with uveitis. Ocul Immunol Inflamm. 2014;22(6):478-84.

39. Psilas K, Aspiotis M, Petroutsos G, Kalogeropoulos C, Constantopoulos S. Antituberculosis therapy in the treatment of peripheral uveitis. AnnOphthalmol. 1991;23(7):254-8. 
